\title{
Baroreflex Control of Plasma Norepinephrine and Heart Period in Healthy Subjects and Diabetic Patients
}

Dwain L. Eckberg, Stephen W. Harkins, Janice M. Fritsch, Gary E. Musgrave, and David F. Gardner Departments of Medicine, Physiology and Biophysics, and Gerontology, Veterans Administration Medical Center and Medical College of Virginia, Richmond, Virginia 23249

\begin{abstract}
Resting diabetic patients may have excessively rapid heart rates, reduced heart rate variability, and subnormal plasma catecholamine levels. Although all of these abnormalities may relate in some way to baroreceptor reflex function, there have been surprisingly few attempts to evaluate systematically baroreflex mechanisms in diabetic patients. Accordingly, we studied autonomic responses over a range of pharmacologically induced arterial pressure changes in 10 unselected young adult insulindependent diabetic patients who had no symptoms of autonomic neuropathy, and 12 age-matched nondiabetic subjects. Sympathetic responses were estimated from antecubital vein plasma norepinephrine levels, and parasympathetic responses were estimated from electrocardiographic $R-R$ intervals and their variability (standard deviation). Both were correlated with other noninvasive indexes of peripheral and central nervous system function. Multiple derangements of baroreflex function were found in the diabetic patients studied. Sympathetic abnormalities included subnormal baseline norepinephrine levels, virtual absence of changes of norepinephrine levels during changes of arterial pressure, and supranormal pressor responses to phenylephrine infusions. Parasympathetic abnormalities included subnormal baseline standard deviations of $\mathbf{R}-\mathbf{R}$ intervals, and $\mathbf{R}-\mathbf{R}$ interval prolongations during elevations of arterial pressure which were unmistakably present, but subnormal. Our data suggest that in diabetic patients, subnormal baseline plasma norepinephrine levels may signify profound, possibly structural defects of sympathetic pathways. Subnormal resting levels of respiratory sinus arrhythmia may have different implications, however, since vagal, unlike sympathetic reflex abnormalities, can be reversed partly by arterial pressure elevations.
\end{abstract}

\section{Introduction}

Subtle or profound abnormalities of autonomic cardiovascular control are found in substantial numbers of diabetic patients (1-12). In this population, resting tachycardia and subnormal heart rate variability point towards vagal neuropathy $(1,2,4-$ $6,10,11)$, and subnormal plasma catecholamine levels point towards sympathetic neuropathy $(3,5,7)$. Published surveys of autonomic function in diabetic patients tend to focus upon

Dr. Eckberg is a Medical Investigator with the Veterans Administration. Dr. Musgrave's current address is Management and Technical Services Company, 600 Maryland Avenue, S.W., Washington, DC 20024.

Address correspondence to Dr. Eckberg, Cardiovascular Physiology, Hunter Holmes McGuire Veterans Administration Medical Center, 1201 Broad Rock Blvd., Richmond, VA 23249.

Received for publication 14 November 1984 and in revised form 5 February 1986

The Journal of Clinical Investigation, Inc

Volume 78, August 1986, 366-374 baseline measurements; there have been surprisingly few attempts to determine how these measurements change in response to changes of autonomic input. Accordingly, we studied autonomic function in healthy, young adult nondiabetic volunteers and age-matched, unselected diabetic patients who had no symptoms of autonomic dysfunction. We estimated sympathetic responses from antecubital vein plasma norepinephrine levels $(13,14)$ and parasympathetic responses from $R-R$ intervals and standard deviations of $R-R$ intervals $(15,16)$, over a range of arterial pressures provoked by stepwise infusions of nitroprusside or phenylephrine. Our findings suggest that sympathetic and vagal abnormalities occur commonly in young diabetic patients, and that these abnormalities may have important pathophysiologic differences.

\section{Methods}

Subjects. All volunteers gave written consent to participate in the research. Nondiabetic subjects comprised seven women and five men whose average age was 26 (range, 21-34) yr. They were healthy, normotensive, and on no medications. Diabetic patients comprised seven women and three men whose average age was 28 (range, 20-36) yr. These patients had had diabetes for an average of 11 (range, 5-17) yr and were treated with insulin (average dose, 45 [range, 24-85] U/d). Patients were recruited from local private, Veterans hospital, and university practices. The only criteria used in their selection were that they be below $37 \mathrm{yr}$ of age and insulin-dependent. Diabetic patients were studied on their usual diets. All studies were begun in the early afternoon after subjects had eaten lunch. Six patients were treated with insulin in divided doses; in these patients, studies were begun 4-5 $\mathrm{h}$ after the first dose, before the second dose was given. Diabetic patients were comparable to control subjects in age, height, and weight. No diabetic patient was obese.

Some clinical characteristics of the diabetic patients are given in Table I. Several patients had apparent complications of diabetes (including absent knee or ankle jerks, six patients; proteinuria, one patient; retinopathy, three patients; and absent dorsal pedal or posterior tibial pulses, six patients). One patient complained of impotence and another had experienced transient nausea and vomiting which subsided spontaneously without treatment. There were no other symptoms attributable to autonomic neuropathy; specifically, no patient had orthostatic hypotension, constipation, nocturnal diarrhea, gustatory sweating, or bladder dysfunction.

Measurements. Studies were conducted with subjects lying supine in a warm, quiet, darkened room. R-R intervals and their standard deviations were calculated from lead II of the electrocardiogram. Tidal volume and breathing frequency were measured with a Respitrace Respiration Monitor (Non-Invasive Monitoring Systems, Miami, FL). Arterial pressure was measured repeatedly by one person with a sphygmomanometer or an ultrasonic device (Arteriosonde 1225, Roche Medical Electronics, Cranbury, NJ). Results were recorded on electrostatic and FM recorders.

Plasma norepinephrine levels were measured with high performance liquid chromatography and electrochemical detection, as described by Hjemdahl and associates (17) and modified by Althaus and co-workers (18). Blood samples were drawn through indwelling antecubital vein needles into chilled tubes containing disodium ethylenediamine tetra- 
acetate and reduced glutathione. These tubes were placed on ice for 10 min or less, and centrifuged at $2,000 \mathrm{rpm}$ and $4^{\circ} \mathrm{C}$, for $10 \mathrm{~min}$. Plasma was then separated and stored at $-70^{\circ} \mathrm{C}$ for $2-17$ wk until analyses were performed. All norepinephrine assays were begun and completed within one 4-wk period. In our laboratory, this method detects norepinephrine levels as low as $10 \mathrm{pg} / \mathrm{ml}$, with a coefficient of variation of $5.6 \%$. Hemoglobin $A_{1}$ levels were measured spectrophotometrically with a commercial kit (Quik-Sep; Isolab, Inc., Akron, $\mathrm{OH}$ ) based on a method developed by Schroeder and co-workers (19). This measurement provides a reliable indication of the long-term quality of diabetic control (20).

Electrophysiologic techniques. The electrophysiologic techniques we used are standard (21). Hearing was normal in all diabetic patients studied. Brain stem auditory-evoked potentials were elicited with 10/s condensation phase clicks presented to the right ear via earphones. Electroencephalographic potentials were recorded between the vertex and the right mastoid process. An ear clip electrode was used as ground.

Somatosensory-evoked potentials were elicited with $3 / \mathrm{s}, 0.5 \mathrm{~ms}$ constant current shocks delivered transcutaneously over the median nerve at the wrist. Shock intensity was set to produce thumb twitches without discomfort. The anode was placed $\sim 2 \mathrm{~cm}$ distal to the cathode, and the ground was placed midway between the elbow and the wrist on the volar surface. Potentials were recorded between electrodes placed parasagitally, $1 \mathrm{~cm}$ inferior and $1 \mathrm{~cm}$ posterior to $\mathrm{C} 4$ (in the 10-20 system [21]), and over the sixth cervical vertebra. Median nerve potentials were recorded from an electrode placed over the median nerve at the elbow.

Electrophysiologic signals were amplified with a Grass P511J amplifier (Grass Instrument Co., Quincy, MA) and averaged with a Nicolet 1074 Signal Summator (Nicolet Instrument Corp., Madison, WI). Evoked potentials were averaged after 1,024 stimuli with 40 or $80 \mu \mathrm{s}$ bins, and median nerve responses were averaged after 64 stimuli. Brain stem latencies were measured between components I and V, and somatosensory latencies were measured between waves P10 and P14. An index of median nerve conduction velocity was obtained by dividing median nerve latencies by the distance between stimulating and recording electrodes.

Acceptable evoked potentials could not be obtained in one nondiabetic volunteer, and one nondiabetic and one diabetic volunteer refused to participate in electrophysiologic testing. In 19 of the remaining 20 volunteers, measurements of autonomic cardiovascular control and of peripheral and central nervous system conduction were made on two separate occasions 7 (range, 0-32) d apart; in one diabetic subject the two studies were conducted $204 \mathrm{~d}$ apart.

Protocol. After the protocol was explained, the volunteer was fitted with electrocardiogram electrodes, respiration monitor, and bilateral antecubital vein butterfly needles. The subject was isolated from investigators and recording equipment by a curtain. There was no talking during experiments. $R-R$ intervals and their standard deviations were calculated during two 4-min periods every $10 \mathrm{~min}$; only measurements obtained during the second 4-min period are reported. Arterial pressure was measured every $2 \mathrm{~min}$; the pressures reported are the averages of the last two pressures during the second 4-min period. In two healthy subjects and one diabetic patient, sinus arrest occurred during phenylephrine infusions. In these subjects, periods of measurement of $R-R$ intervals were shortened to include only the period of sinus rhythm before the onset of sinus arrest. In the two nondiabetic subjects, five periods of measurement were less than $4 \mathrm{~min}$, and averaged 126 and $62 \mathrm{~s}$. In the diabetic patient, seven periods of measurement were less than $4 \mathrm{~min}$; these averaged $121 \mathrm{~s}$. Blood samples were drawn at the end of each 10-min period.

Experiments began with a 30-min rest period during which normal saline was infused at a rate of $100 \mu \mathrm{l} / \mathrm{min}$. The last measurements obtained during this 30 -min period were used as control values. Then, infusions of sodium nitroprusside or phenylephrine hydrochloride were begun. Choice of drug sequence was dictated by a coin toss (in practice, seven nondiabetic and five diabetic subjects were given phenylephrine first). Both drugs were given as sequential, stepwise, 10-min infusions in concentrations of $0.2,0.4,0.8,1.6$, and (for nitroprusside) $3.2 \mu \mathrm{g} / \mathrm{kg}$ per min. Infusions were stopped after diastolic arterial pressure rose or fell by $\sim 15 \mathrm{mmHg}$, the maximum planned dose was given, symptoms supervened, or cardiac rhythm changed. (Two nondiabetic subjects and one diabetic patient developed premature ventricular beats during phenylephrine infusions.)

When the first series of drug infusions was completed, saline was infused for a second 30-min period. In one diabetic patient who was given phenylephrine first, this period was extended to $50 \mathrm{~min}$ because his blood pressure did not return to control levels during the usual 30min period. Following this, infusions of the second vasoactive drug were begun. The last measurements obtained during the second control period were used as baseline values for responses to the second vasoactive drug. Diastolic pressures are emphasized in this report, because they correlate more closely with sympathetic activity than systolic pressures (22).

Statistical analyses. Data are presented as mean values \pm SEM. The Kolmogorov-Smirnov test was used to evaluate the distribution of data. Because many data sets were not distributed normally, nonparametric statistical comparisons were made (23). Intragroup correlations between measurements were sought with the Kendall Tau B statistic, and intragroup differences between responses were sought with the Wilcoxon signed rank test. Intergroup comparisons were made with the Mann-WhitneyWilcoxon distribution. Differences were considered significant when $P$ $\leq 0.05$. When measurements could be predicted on the basis of published data, one-tailed tests were used.

\section{Results}

Baseline measurements. Clinical data for diabetic patients and baseline measurements for control subjects and diabetic patients are given in Tables I and II and Figs. 1 and 2. Resting arterial pressures were comparable in nondiabetic and diabetic subjects. In all volunteers, measurements of arterial pressure were highly reproducible; for example, in nondiabetic subjects, the last six systolic pressures measured during the first saline infusion averaged $115 \pm 4,116 \pm 3,115 \pm 2,114 \pm 2,115 \pm 3$, and $114 \pm 2$ $\mathrm{mmHg}$.

Average $R-R$ intervals and standard deviations of $R-R$ intervals for all subjects are shown in Fig. 1, and are given in Table II. Average baseline R-R intervals were insignificantly higher in nondiabetic than diabetic subjects $(0.950 \pm 0.046$ vs. $0.870 \pm 0.051$ $\mathrm{s}, P=0.065$ ). Average baseline standard deviations of $\mathrm{R}-\mathrm{R}$ intervals (measures that appear to correlate well with levels of efferent vagal-cardiac nerve traffic $[15,16,24])$ were significantly higher in nondiabetic than diabetic subjects $(0.068 \pm 0.005$ vs. $0.048 \pm 0.010 \mathrm{~s}, P=0.010$ ). Six diabetic patients had average control standard deviations of $R-R$ intervals below the lowest value obtained from nondiabetic subjects.

Mean peak-valley heart rate changes during single deep breaths, a measure used by others (10) to diagnose disordered vagal-cardiac control in diabetic patients, also were significantly different in nondiabetic and diabetic subjects (Table II). Average sighs were insignificantly smaller in nondiabetic than diabetic subjects ( $1.90 \pm 0.30$ vs. $2.50 \pm 0.351, P=0.145$, two-tailed test), but average peak-valley changes of heart rate were significantly larger ( $26 \pm 3$ vs. $14 \pm 3$ beats $/ \mathrm{min}, P=0.005$ ). The average heart rate change during deep breaths in diabetic patients was influenced importantly by a change of 34 beats/min that occurred in one diabetic patient. Baseline respiratory rates and depths were comparable in nondiabetic and diabetic volunteers. The average change of mean blood pressure after $3 \mathrm{~min}$ of standing (in nine diabetic patients) was -0.4 (range, 9 to -7 ) $\mathrm{mmHg}$. No patient experienced orthostatic symptoms during standing.

Average baseline antecubital vein plasma norepinephrine levels for all subjects are shown in Fig. 2 and are given in Table II. In nondiabetic subjects, plasma norepinephrine averaged 228 (range, 60-441; SD, 133) pg/ml. In diabetic patients, baseline plasma norepinephrine levels averaged 119 (range, 13-388; SD, 
Table I. Clinical Characteristics of Patients

\begin{tabular}{|c|c|c|c|c|c|c|c|c|}
\hline & $\begin{array}{l}\text { Patient } \\
\text { number }\end{array}$ & Sex & Age & $\begin{array}{l}\text { Duration of } \\
\text { diabetes }\end{array}$ & Weight & Blood pressure & $\begin{array}{l}\text { Change mean blood } \\
\text { pressure, standing }\end{array}$ & Hemoglobin $A_{1}$ \\
\hline & & & $y r$ & $y r$ & $k g$ & $m m H g$ & $m m H g$ & percent \\
\hline & 1 & $\mathrm{~F}$ & 32 & 17 & 56.8 & $105 / 71$ & -6 & 16 \\
\hline & 2 & $\mathrm{~F}$ & 23 & 16 & 63.6 & $116 / 75$ & - & 9 \\
\hline & 3 & $F$ & 26 & 8 & 54.5 & $113 / 85$ & 0 & 12 \\
\hline & 4 & $\mathrm{~F}$ & 20 & 8 & 65.0 & $118 / 71$ & 9 & 10 \\
\hline & 5 & $\mathbf{M}$ & 36 & 5 & 74.1 & $104 / 66$ & 8 & 10 \\
\hline & 6 & $\mathbf{M}$ & 27 & 7 & 78.2 & $99 / 65$ & -7 & 14 \\
\hline & 7 & $\mathrm{~F}$ & 24 & 10 & 58.6 & $101 / 78$ & -5 & 11 \\
\hline & 8 & $\mathbf{M}$ & 32 & 13 & 72.7 & $129 / 89$ & 0 & 16 \\
\hline & 9 & $F$ & 23 & 6 & 60.5 & $107 / 71$ & -2 & 19 \\
\hline & 10 & $\mathrm{~F}$ & 35 & 16 & 56.8 & $99 / 63$ & -1 & 11 \\
\hline Mean & & & 28 & 10.6 & 64.1 & $109 / 73$ & -0.4 & 12.8 \\
\hline SEM & & & 2 & 1.4 & 2.6 & $3 / 3$ & 1.9 & 1.0 \\
\hline \multicolumn{9}{|c|}{ Control subjects } \\
\hline Mean & & $7 \mathrm{~F} / 5 \mathrm{M}$ & 26 & - & 67.8 & $113 / 72$ & & 6.8 \\
\hline SEM & & & 4 & - & 3.2 & $2 / 2$ & & 0.4 \\
\hline \multicolumn{9}{|l|}{$\begin{array}{r}P \text { (diabetic vs. } \\
\text { nondiabetic }\end{array}$} \\
\hline volunteers) & & 0.592 & 0.322 & & 0.387 & $0.131 / 0.425$ & & $<0.001$ \\
\hline
\end{tabular}

106) $\mathrm{pg} / \mathrm{ml}$, and were significantly $(P=0.009$, two-tailed test) lower than those measured in nondiabetic subjects. Two diabetic patients had control norepinephrine levels below the lowest level measured in nondiabetic subjects. As expected, hemoglobin $A_{1}$ levels were significantly $(P<0.001)$ lower in nondiabetic than diabetic subjects.

Average latencies between waves I and $\mathrm{V}$ of brain stem auditory-evoked potentials were significantly shorter $(P=0.001)$ in nondiabetic than diabetic subjects (Table II). The I-V latency was not related significantly to the duration of diabetes or to the hemoglobin $\mathrm{A}_{1}$ levels $(P=0.375$ each). The average median nerve conduction velocity was significantly $(P=0.004)$ more rapid in nondiabetic than diabetic patients. Median nerve conduction velocity in diabetic patients was not related to the duration of diabetes $(P=0.191)$, but was related inversely to hemoglobin $A_{1}$ levels $(P=0.039)$. Average somatosensory-evoked potential interpeak latencies were comparable in diabetic and nondiabetic volunteers $(P=0.407$, two-tailed test).

Responses to nitroprusside. Nitroprusside infusions caused significant reductions of systolic and diastolic arterial pressures in both groups. Average maximum doses of nitroprusside were similar in nondiabetic and diabetic subjects. Average maximum reductions of diastolic pressures were insignificantly less in nondiabetic than diabetic subjects ( $12 \pm 3$ vs. $15 \pm 4 \mathrm{mmHg}, P=0.288$, two-tailed test). Average $R-R$ interval responses to nitroprusside are shown in Fig. 3, left. Average maximum reductions of R-R intervals and $R-R$ interval changes expressed as functions of diastolic pressure changes, were comparable in the two groups ( $P=0.359$ and $P=0.134$, two-tailed tests).

In nondiabetic subjects, changes of plasma norepinephrine levels during nitroprusside infusions were complex (Fig. 4, left). As the rate of nitroprusside infusion was increased to 1.6 and $3.2 \mu \mathrm{g} / \mathrm{kg}$ per min, plasma norepinephrine levels declined to $122 \pm 71$ and $49 \pm 115 \mathrm{pg} / \mathrm{ml}$ above baseline levels. A reduction of plasma norepinephrine concentration from the preceding level occurred with the highest rate of nitroprusside infusion in all but three subjects. Statistical comparisons of plasma norepinephrine levels during vasoactive drug infusions in nondiabetic subjects are given in Table III.

In diabetic patients (Fig. 4, right), average norepinephrine concentrations increased slightly, but insignificantly, during infusions of nitroprusside. The highest average plasma norepinephrine level was influenced importantly by increases of 191 and $195 \mathrm{pg} / \mathrm{ml}$ that occurred in two patients (Table II). The maximum increase of plasma norepinephrine levels during nitroprusside infusions was significantly larger in nondiabetic than diabetic subjects $(146 \pm 75$ vs. $36 \pm 34 \mathrm{pg} / \mathrm{ml}, P=0.012$, twotailed test).

Responses to phenylephrine. Phenylephrine infusions caused significant increases of systolic and diastolic arterial pressures in both groups. The average maximum rate of infusion of phenylephrine given to nondiabetic subjects was significantly larger than that given to diabetic subjects $(1.47 \pm 0.09$ vs. $1.00 \pm 0.17$ $\mu \mathrm{g} / \mathrm{kg}$ per min, $P=0.030$, two-tailed test), but changes of diastolic pressure were comparable ( $P=0.105$, two-tailed test).

Responses of one diabetic patient to phenylephrine (and nitroprusside) infusions are shown in Fig. 5. In this patient, the baseline standard deviation of R-R interval was lower than 2 $\mathrm{SD}$ from the mean obtained in nondiabetic subjects; however, phenylephrine infusion (Fig. 5, right) provoked a substantial prolongation of $R-R$ intervals and an increase of the standard deviation of $R-R$ intervals. Average changes of $R-R$ intervals during phenylephrine infusions for all subjects are shown in Fig. 3, right. Maximum R-R interval prolongations, and R-R interval prolongations expressed as functions of diastolic pressure changes were significantly greater in nondiabetic than diabetic subjects ( $P=0.006$ and $P=0.001$, two-tailed tests).

Average maximum reductions of plasma norepinephrine levels during phenylephrine infusions (Fig. 4) were significantly greater in nondiabetic than diabetic volunteers $(100 \pm 28$ vs. $2 \pm 28$ 


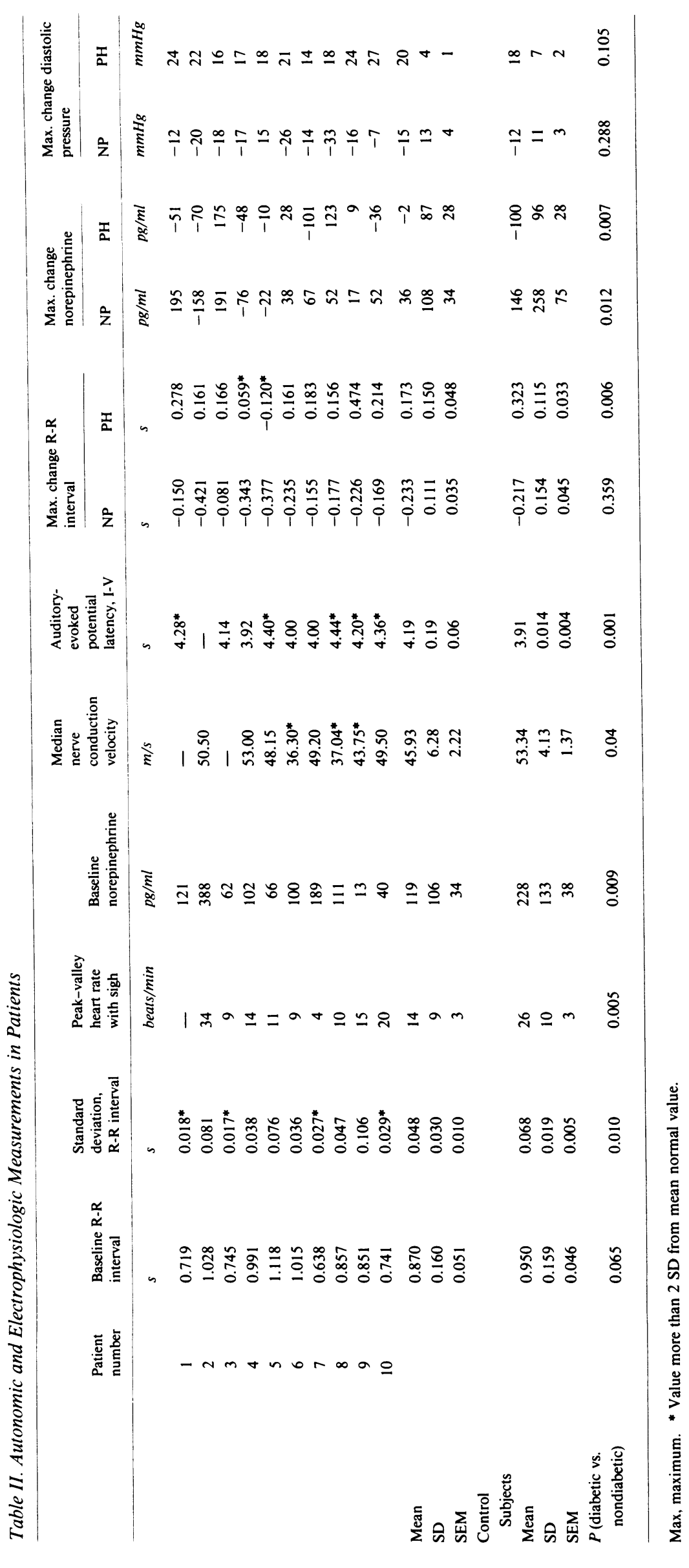



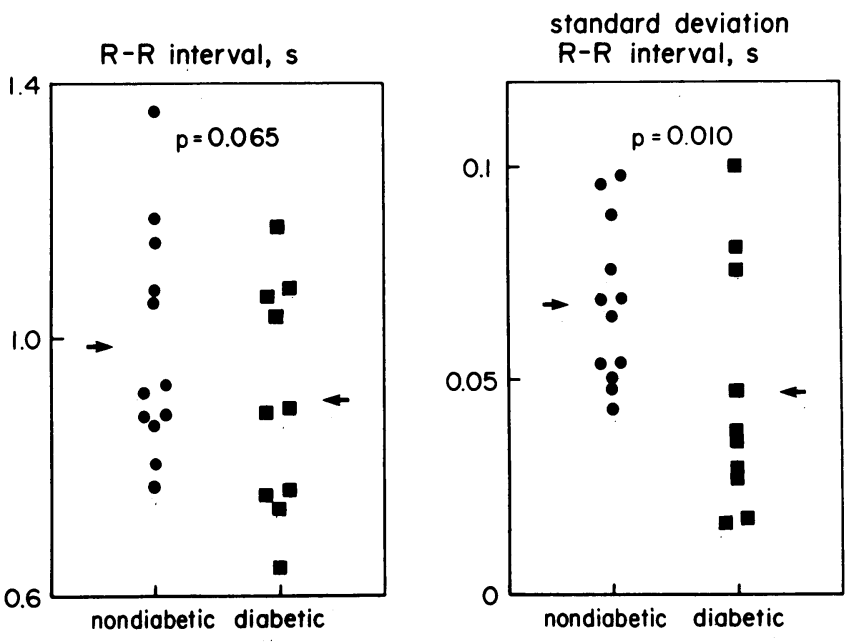

Figure 1. Baseline R-R intervals and standard deviations of R-R intervals for all subjects. Arrows indicate average values for each group. $P$ values were derived with the Mann-Whitney-Wilcoxon distribution (23).

$\mathrm{pg} / \mathrm{ml}, P=0.007$, two-tailed test). Norepinephrine levels under baseline conditions in diabetic patients were comparable to levels after maximum baroreflex suppression in nondiabetic subjects $(P=0.140$, two-tailed test). The average highest levels of plasma norepinephrine during nitroprusside infusions, and lowest levels of plasma norepinephrine during phenylephrine infusions are shown with their corresponding diastolic pressures in Fig. 6.

\section{Discussion}

We obtained indirect measurements of sympathetic and vagalcardiac nervous activity over a range of arterial pressures in healthy young adult volunteers and diabetic patients who had no clinical evidence for autonomic neuropathy. Baroreflex measurements were correlated with other measurements of peripheral and central nervous system function and with the quality of

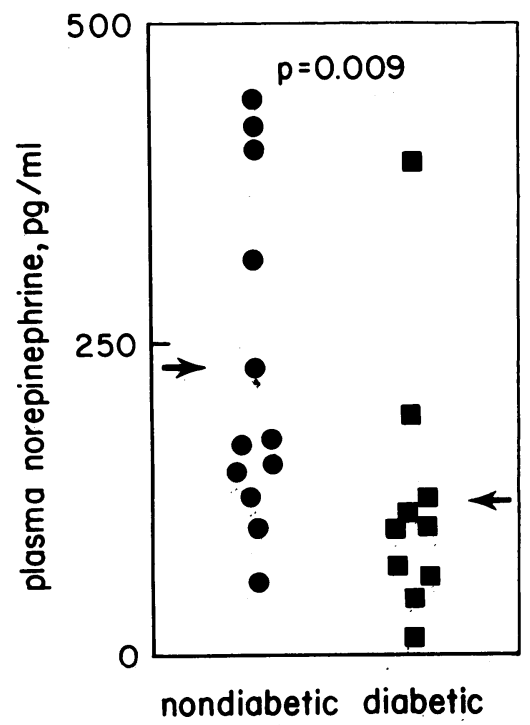

Figure 2. Average baseline antecubital vein plasma norepinephrine levels for all subjects.

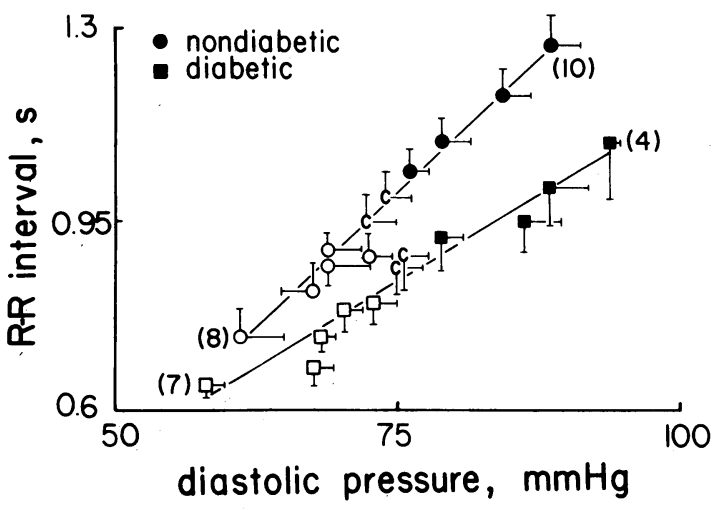

Figure 3. Average R-R intervals and corresponding diastolic pressures during infusions of vasoactive drugs for all subjects. Open circles and squares indicate responses to nitroprusside, and closed circles and squares indicate responses to phenylephrine. $C$, control measurements before nitroprusside or phenylephrine. Brackets encompass 1 SEM.

Numbers in parentheses indicate the numbers of patients receiving the highest doses. Lines were drawn according to best visual fit. Phenylephrine responses (closed circles and squares) were significantly $(P$ $=0.001$, Mann-Whitney-Wilcoxon distribution [23], two-tailed test) greater in nondiabetic than diabetic subjects.

diabetic control. Our observations may provide new insights into both physiologic and pathophysiologic mechanisms of autonomic cardiovascular regulation.

Physiologic mechanisms. Antecubital vein plasma norepinephrine levels correlate significantly with resting levels of directly measured postganglionic muscle sympathetic nerve activity (25), and during pharmacologic changes of arterial pressure (Eckberg, D. L., O. K. Andersson, T. Hedner, J. M. Lundberg, R. F. Rea, and B. G. Wallin, unpublished observations). Therefore, our findings provide some quantitative estimate of how baroreceptor inputs (arterial and cardiopulmonary) determine sympathetic activity. The principal conclusions drawn from the responses of healthy nondiabetic subjects are that baroreflex modulation of sympathetic activity is complex and assymetric (Fig. 4, left).

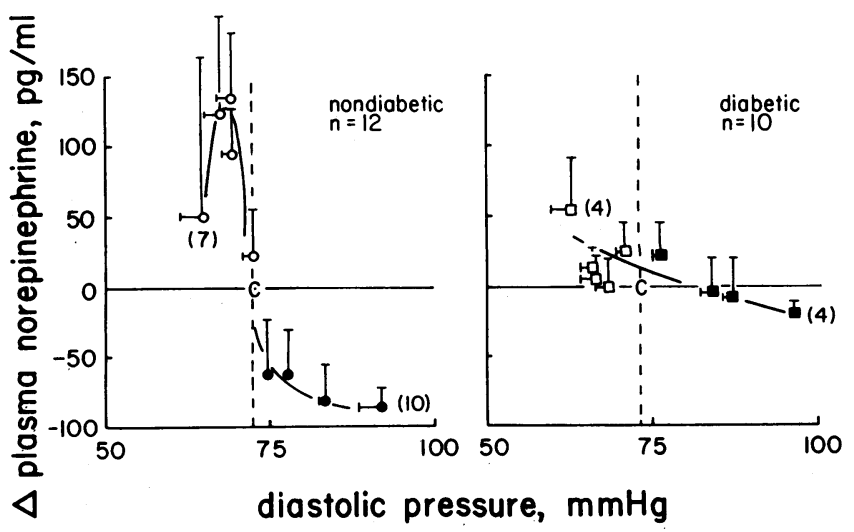

Figure 4. Sympathetic responses to changes of arterial pressure. Since changes of blood pressure were proportional to rates of infusions of vasoactive drugs, successive points to the left or right of control values represent responses to increasing doses of nitroprusside or phenylephrine. Sample sizes at the lowest diastolic pressures are smaller than those in Fig. 3 because in some experiments, infusions of the largest doses of vasoactive drugs were ended before $8 \mathrm{~min}$ had elapsed. 
Table III. Norepinephrine Responses to Vasoactive Drugs

\begin{tabular}{|c|c|c|c|c|c|c|c|}
\hline \multirow{2}{*}{\multicolumn{3}{|c|}{$\begin{array}{l}\text { Nitroprusside dose } \\
\mu \mathrm{g} / \mathrm{kg} \text { per min }\end{array}$}} & \multirow{3}{*}{$\begin{array}{l}P \\
0.151\end{array}$} & \multirow{2}{*}{\multicolumn{3}{|c|}{$\frac{\text { Phenylephrine dose }}{\mu \mathrm{g} / \mathrm{kg} \text { per min }}$}} & \multirow{3}{*}{$\begin{array}{l}P \\
0.017\end{array}$} \\
\hline & & & & & & & \\
\hline \multirow[t]{5}{*}{0} & (Control) vs. & 0.2 & & 0 & (Control) vs. & 0.2 & \\
\hline & & 0.4 & 0.008 & & & 0.4 & 0.001 \\
\hline & & 0.8 & 0.009 & & & 0.8 & 0.001 \\
\hline & & 1.6 & 0.032 & & & 1.6 & 0.001 \\
\hline & & 3.2 & 0.148 & & & & \\
\hline \multirow{4}{*}{\multicolumn{2}{|c|}{0.2}} & 0.4 & 0.001 & 0.2 & & 0.4 & 0.485 \\
\hline & & 0.8 & 0.001 & & & 0.8 & 0.080 \\
\hline & & 1.6 & 0.037 & & & 1.6 & 0.161 \\
\hline & & 3.2 & 0.188 & & & & \\
\hline \multirow{3}{*}{\multicolumn{2}{|c|}{0.4}} & 0.8 & 0.116 & 0.4 & & 0.8 & 0.120 \\
\hline & & 1.6 & 0.385 & & & 1.6 & 0.348 \\
\hline & & 3.2 & 0.234 & & & & \\
\hline \multirow{2}{*}{\multicolumn{2}{|c|}{0.8}} & 1.6 & 0.423 & 0.8 & & 1.6 & 0.500 \\
\hline & & 3.2 & 0.109 & & & & \\
\hline 1.6 & & 3.2 & 0.078 & & & & \\
\hline
\end{tabular}

Statistical comparisons (Wilcoxon signed rank test [23]) between plasma norepinephrine levels at different doses during infusions of nitroprusside and phenylephrine, in nondiabetic subjects. Average plasma norepinephrine levels tended to decline as the phenylephrine infusion rate was increased (Fig. 4, left, closed circles). This statistical analysis suggests that although the higher doses of phenylephrine (presumably associated with higher levels of baroreceptor stimulation) led to greater reductions of plasma norepinephrine levels than the initial, very low dose, the decrement of sympathetic activity at these higher doses was small.

First, we found that reductions of plasma norepinephrine levels during elevations of arterial pressure are less than elevations of plasma norepinephrine during reductions of arterial pressure.
This suggests that sympathetic mechanisms are more important in opposing arterial pressure reductions than they are in preventing arterial pressure elevations. Second, the ability of sympathetic mechanisms to buffer reductions of arterial pressure is limited by the extent of increased sympathetic activity, and by the biphasic pattern of sympathetic responses. The highest norepinephrine levels we measured during arterial pressure reductions were considerably lower than those measured by others during other stresses, such as upright posture and treadmill exercise (26). Moreover, although small reductions of arterial pressure increase plasma norepinephrine levels, larger reductions of arterial pressure return plasma norepinephrine towards baseline levels.

The observation that reductions of sympathetic activity occur systematically in man as blood pressure falls from mild to moderate levels (Fig. 4, left) was consistent (it occurred in 9 of 12 nondiabetic subjects), is new, and is of uncertain significance. One interpretation is that as arterial pressure falls, other mechanisms (such as increases of afferent traffic from left ventricular receptors [27]) are activated which oppose baroreflex adjustments. Hypotension, provoked by progressive hemorrhage (28), lower body negative pressure (29), or nitroprusside infusion (30) in human volunteers may lead to sudden reversal of heart rate, from tachycardia to bradycardia, and sympathetic activity, from higher to lower levels. Alternatively, reductions of plasma norepinephrine levels during the highest rate of nitroprusside infusion may reflect acute resetting of baroreflexes (31), or it may be due merely to increased washout of norepinephrine secondary to increased forearm blood flow.

Systematic reductions of plasma norepinephrine levels during infusions of nitroprusside were not found by Lin, McNay, Shepherd, and Keeton (32) who studied a smaller population $(n=5)$ of patients with hypertension, and used different doses of nitroprusside than we used. However, the pattern of plasma norepinephrine changes we observed in human subjects is very similar to the pattern of renal sympathetic nerve changes observed by Dorward and co-workers (33) during hypotension in conscious rabbits.

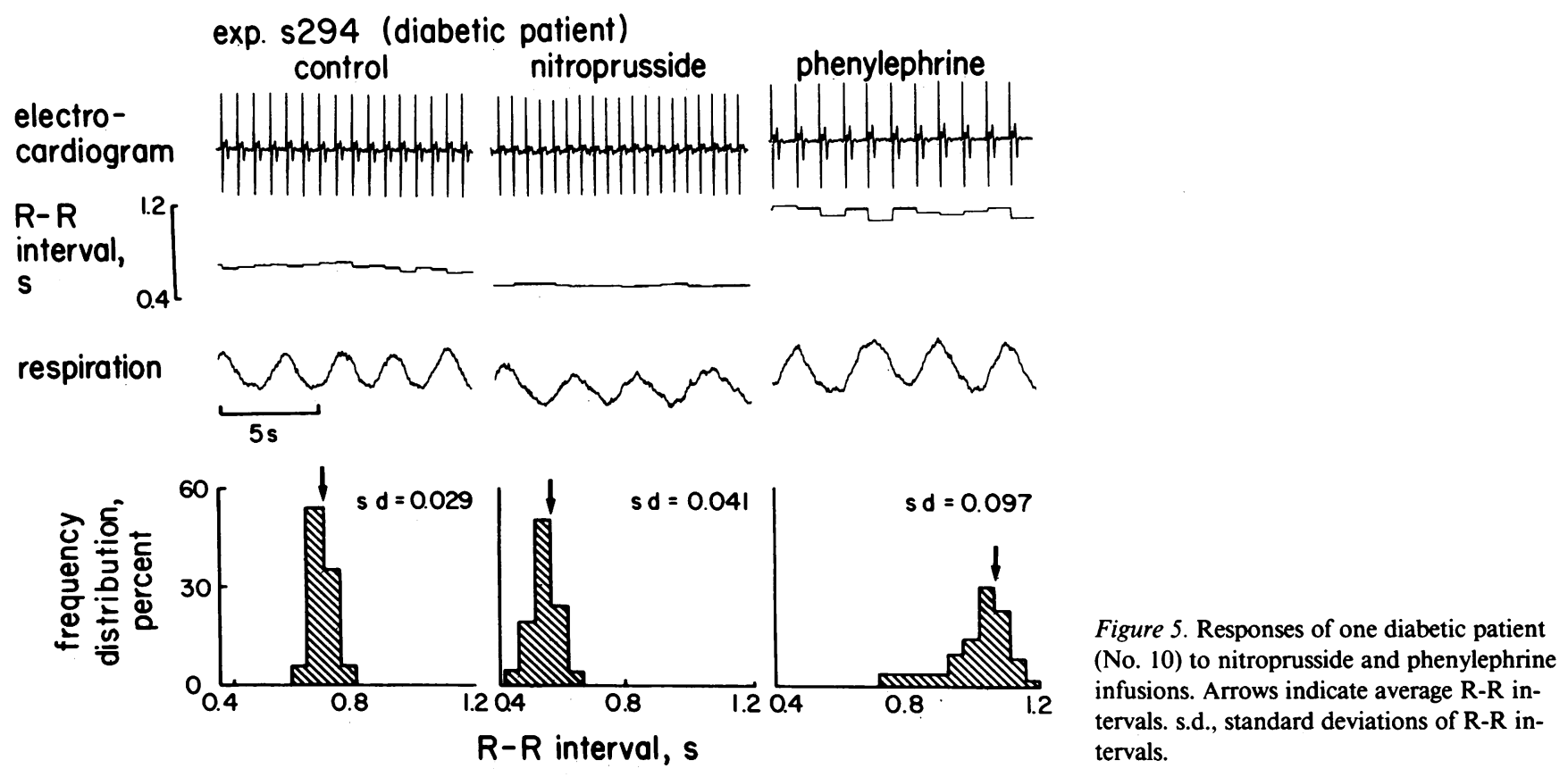




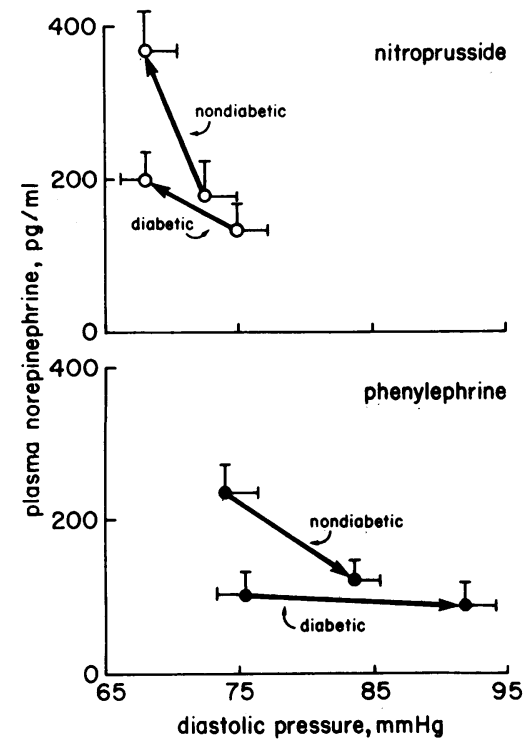

Figure 6. Maximum increases and decreases of plasma norepinephrine levels and corresponding diastolic pressures during nitroprusside and phenylephrine infusions. The arrows indicate the directions of changes from control values. Maximum reductions and increases of diastolic pressures during nitroprusside and phenylephrine infusions were insignificantly $(P=0.288$ and $P=0.105)$ greater, but increases and reductions of plasma norepinephrine levels were significantly $(P=0.012$ and $P=0.007$ ) less in diabetic than nondiabetic volunteers. The moderate rise of the average maximum norepinephrine level during nitroprusside infusions in diabetic patients resulted primarily from increases of 191 and $195 \mathrm{pg} / \mathrm{ml}$ that occurred in two patients.

Pathophysiologic mechanisms. The baroreflex responses of the unselected young adult, insulin-dependent diabetic patients we studied provided compelling evidence for abnormalities of both sympathetic and vagal cardiovascular control. Baseline antecubital vein plasma norepinephrine levels were subnormal. In the diabetic patients we studied, who (with the possible exception of one man with impotence) did not have symptoms of autonomic dysfunction, low resting plasma norepinephrine levels signified profound disturbances of sympathetic control: with the exception of two patients, there was no credible evidence that sympathetic outflow was modulated by changes of arterial pressure.

Resting vagal-cardiac outflow, as reflected by average standard deviations of $R-R$ intervals, also was subnormal in these diabetic patients; however, this abnormality was quantitative, rather than qualitative, since vagal-cardiac activity could be restored towards normal levels by elevations of arterial pressure. We suspect that although abnormalities of sympathetic and parasympathetic cardiovascular control may reflect a generalized neuropathy in diabetic patients (34; and this study), they may have substantially different pathophysiologic bases.

Sympathetic abnormalities. Subnormal baseline plasma norepinephrine levels have been found in diabetic patients previously, but usually in subsets of patients selected because they had other evidence for neuropathy $(3,5,7,35,36)$. For example, Christensen (7) found subnormal total plasma catecholamine levels at rest in a group of nine diabetic patients who had diminished vibratory sensation, but Cryer, Silverberg, Santiago, and Shah (37) found normal average norepinephrine levels in a large group of patients who were unselected. We cannot explain fully the disparity between Cryer's findings and our own. The average duration of diabetes was comparable in the two studies, but Cryer's patients tended to be older, heavier, and less likely to be taking insulin than ours. Also, Cryer drew samples for baseline norepinephrine determinations in the morning rather than at noon as we did.

However, we believe that internal evidence supports the trustworthiness of our data. The average norepinephrine level we obtained in nondiabetic subjects was virtually identical to average levels measured by others (including Cryer et al. [37]) under similar circumstances (13). Moreover, the depressed baseline norepinephrine levels we measured in diabetic patients were associated with other evidence for sympathetic dysfunction, including lack of baroreflex modulation of norepinephrine levels and supranormal pressor responses to phenylephrine. Our observation that patients with low average resting norepinephrine levels do not modulate their norepinephrine levels as blood pressure changes is new. However, this finding complements earlier observations of others which suggest that diabetic patients who have subnormal resting plasma norepinephrine levels also have subnormal responses to stresses that normally increase sympathetic outflow, including upright posture $(7,36)$, exercise (3), and low levels of lower body negative pressure (6).

We found additional evidence for sympathetic (and probably parasympathetic) neuropathy in the responses of diabetic patients to infusions of the alpha-adrenergic agonist, phenylephrine. In diabetic patients, arterial pressure rose to higher levels after significantly smaller doses of phenylephrine than in nondiabetic subjects. Hypersensitivity to infusions of catecholamines has been recorded before in diabetic patients $(9,38)$, and is likely to be of multifactoral etiology.

First, hypersensitivity to pressors may be due to subnormal baroreflex buffering of blood pressure elevations (Fig. 3), resulting in less heart rate slowing per unit pressure elevation and near absence of withdrawal of sympathetic activity as arterial pressure rises (Fig. 4, right). The absence of significant changes of plasma norepinephrine levels during infusions of vasoactive drugs cannot be explained simply on the basis of absent baroreceptor sensing of pressure changes, however, because sinus node responses in these patients were substantial (Fig. 3, right). Moreover, subnormal baroreceptor-cardiac reflex responses should be associated with heightened, not reduced, resting levels of plasma norepinephrine (39).

Second, enhanced pressor responsiveness may be explained on the basis of supersensitivity to catecholamines associated with reduced numbers of postganglionic sympathetic nerves. Muscle sympathetic nerve activity can be recorded in a larger percentage of nondiabetic than diabetic subjects (8), and diabetic patients may have axonal degeneration and loss of unmyelinated nerves (9), and subnormal concentrations of catecholamines in peripheral arteries (38). Third, enhanced pressor responsiveness to phenylephrine may be due partly to structural abnormalities of resistance vessels (40), since responses to noncatechol vasoactive agents, such as angiotensin II (41) may be greater in diabetic than nondiabetic subjects.

Although the diabetic patients we studied did not have orthostatic hypotension, the abnormal pattern of measurements we found is qualitatively similar to that found by others $(42,43)$ in patients with primary orthostatic hypotension (that is, hypotension secondary to neuropathy of postganglionic sympathetic nerves). In patients with primary orthostatic hypotension, low resting norepinephrine levels and increased responsiveness 
to infused norepinephrine are associated with diminished histochemical catecholamine fluorescence of sympathetic nerves (42).

Parasympathetic abnormalities. Average resting vagal-cardiac activity, as reflected by standard deviations of R-R intervals $(15,16)$, was subnormal in diabetic patients (Fig. 1, right). Subnormal variability of $R-R$ intervals (standard deviations [11, 12], or peak-valley $R-R$ intervals $[2,4]$ ) has been found in diabetic patients before, and has been considered to be due to vagal neuropathy (1). However, we found that average baseline standard deviations do not predict $\mathbf{R}-\mathbf{R}$ interval changes during pressor infusions (Fig. 3). This observation may be related to earlier findings of others which underscore the reversibility of vagalcardiac abnormalities: Bennett et al. found that diabetic patients who have subnormal $R-R$ interval variability at rest may have large R-R interval variability during sleep (11) or face immersion (10). Thus, these and our own findings are not consistent with simple anatomic destruction of vagal-cardiac nerves.

We cannot be certain why the diabetic patients we studied had subnormal levels of vagal-cardiac outflow at rest. However, we offer three possibilities: First, low baseline standard deviations of R-R intervals could be due to subnormal resting levels of stimulation of vagal-cardiac nuclei by baroreceptor input; our findings (Fig. 3, right), and those of others $(5,9)$ show that R-R interval prolongations expressed as functions of arterial pressure increases are subnormal in diabetic patients. Abnormal baroreceptor sensing could explain why vagal responses may be subnormal during increases of arterial baroreceptor input $(5,9$, and this study) but normal during increases of trigeminal nerve input provoked by face immersion (10).

Second, subnormal vagal outflow at rest in diabetic patients may be due to an abnormal central "setting" of the activity of vagal-cardiac motonuclei. This possibility is supported indirectly by the prolongation of average brainstem auditory-evoked potentials we found; although this finding cannot be related causally to vagal dysfunction, it documents the existence of abnormalities of central nervous system function in the diabetic patients we studied. The prolongation of average brainstem auditory-evoked potential latencies we measured is nearly identical to that found by Donald and co-workers (44) who studied a much larger group $(n=50)$ of diabetic patients. The possibility that subnormal resting vagal-cardiac outflow is due to a reversible, abnormal central setting is supported by the finding of Bennett et al. (11) that some diabetic patients with subnormal respiratory variations of heart rate at rest, may have striking increases of respiratory sinus arrhythmia during sleep.

A third potential mechanism, and one which is highly likely, is that resting and provoked levels of vagal-cardiac activity are subnormal because of dysfunction of vagal-cardiac fibers or diminution of their numbers. This possibility is supported inferentially by our documentation of abnormalities of function of other myelinated nerves in the patients we studied (subnormal median nerve conduction velocities and prolonged auditory evoked potential latencies). The possibility also is supported by the histologic study of Duchen, Anjorin, Watkins, and Mackay (45), which shows that small myelinated vagal fibers (not necessarily cardiac) may be diseased or absent in diabetic patients.

In summary, we studied baroreceptor modulation of sympathetic and parasympathetic cardiovascular nervous outflow in young adult nondiabetic and diabetic volunteers. Sympathetic abnormalities found in diabetic patients included subnormal resting levels of plasma norepinephrine, virtual absence of baroreceptor modulation of norepinephrine concentrations, and ex- aggerated pressor responses to infused phenylephrine. This pattern of abnormalities is consistent with destruction of distal postganglionic sympathetic nerves. Vagal abnormalities included subnormal resting $R-R$ interval variability and impaired baroreflex augmentation of $R-R$ intervals. Parasympathetic abnormalities were partially reversible, and may be due to a combination of factors; however, they are unlikely to be due simply to anatomic destruction of efferent vagal-cardiac nerves. Our findings suggest that commonly used baseline measurements may not characterize autonomic function adequately in diabetic patients. Moreover, although our data were not obtained longitudinally, they cast considerable doubt on the dictum (34) that in diabetes, loss of vagal autonomic cardiovascular control precedes loss of sympathetic control.

\section{Acknowledgments}

We thank John J. Schelhorn for his technical assistance, Laura L. Hearne, Dana L. Montague, and E. Jean Ryland for their secretarial help, Vernon M. Chinchilli for his statistical advice, James W. Sowers and Marc D. Thames for their critical reviews of the manuscript, and Dr. George W. Campbell, Dr. David C. Grulke, and Dr. D. H. Zontine for their referral of patients.

This research was supported by grants from the Veterans Administration and the National Institutes of Health (HL 22296, HL 22070, and HL 22546).

\section{References}

1. Murray, A., D. J. Ewing, I. W. Campbell, J. M. M. Neilson, and B. F. Clarke. 1975. RR interval variations in young male diabetics. $B r$. Heart J. 37:882-885.

2. Young, R. J., D. J. Ewing, and B. F. Clarke. 1983. Nerve function and metabolic control in teenage diabetics. Diabetes. 32:142-147.

3. Hilsted, J., H. Galbo, and N. J. Christensen. 1980. Impaired responses of catecholamines, growth hormone, and cortisol to graded exercise in diabetic autonomic neuropathy. Diabetes. 29:257-262.

4. Watkins, P. J., and J. D. Mackay. 1980. Cardiac denervation in diabetic neuropathy. Ann. Intern. Med. 92:304-307.

5. Bennett, T., D. J. Hosking, and J. R. Hampton. 1976. Baroreflex sensitivity and responses to the Valsalva manoeuvre in subjects with diabetes mellitus. J. Neurol. Neurosurg. Psychiatry. 39:178-183.

6. Bennett, T., D. J. Hosking, and J. R. Hampton. 1980. Cardiovascular responses to graded reductions of central blood volume in normal subjects and in patients with diabetes mellitus. Clin. Sci. 58:193-200.

7. Christensen, N. J. 1972. Plasma catecholamines in long-term diabetics with and without neuropathy and in hypophysectomized subjects. J. Clin. Invest. 51:779-787.

8. Fagius, J., and B. G. Wallin. 1980. Sympathetic reflex latencies and conduction velocities in patients with polyneuropathy. J. Neurol. Sci. 47:449-461.

9. Low, P. A., J. C. Walsh, C. Y. Huang, and J. G. McLeod. 1975. The sympathetic nervous system in diabetic neuropathy. A clinical and pathological study. Brain. 98:341-356.

10. Bennett, T., I. K. Farquhar, D. J. Hosking, and J. R. Hampton. 1978. Assessment of methods for estimating autonomic nervous control of the heart in patients with diabetes mellitus. Diabetes. 27:1167-1174.

11. Bennett, T., P. H. Fentem, D. Fitton, J. R. Hampton, D. J. Hosking, and P. A. Riggott. 1977. Assessment of vagal control of the heart in diabetes. Measures of $R-R$ interval variation under different conditions. Br. Heart J. 39:25-28.

12. Ewing, D. J., D. Q. Borsey, F. Bellavere, and B. F. Clarke. 1981. Cardiac autonomic neuropathy in diabetes: comparison of measures of R-R interval variation. Diabetologia. 21:18-24.

13. Goldstein, D. S., R. McCarty, R. J. Polinsky, and I. J. Kopin. 1983. Relationship between plasma norepinephrine and sympathetic neural activity. Hypertension. 5:552-559. 
14. Grossman, S. H., D. Davis, J. C. Gunnells, and D. G. Shand. 1982. Plasma norepinephrine in the evaluation of baroreceptor function in humans. Hypertension. 4:566-571.

15. Eckberg, D. L. 1983. Human sinus arrhythmia as an index of vagal cardiac outflow. J. Appl. Physiol. 54:961-966.

16. Katona, P. G., and F. Jih. 1975. Respiratory sinus arrhythmia: noninvasive measure of parasympathetic cardiac control. J. Appl. Physiol. 39:801-805.

17. Hjemdahl, P., M. Daleskog, and T. Kahan. 1979. Determination of plasma catecholamines by high performance liquid chromatography with electrochemical detection: comparison with a radioenzymatic method. Life Sci. 25:131-138.

18. Miller, E. D., Jr., J. J. Beckman, J. R. Woodside, Jr., J. S. Althaus, and M. J. Peach. 1983. Blood pressure control during anesthesia: importance of the peripheral sympathetic nervous system and renin. Anesthesiology. 58:32-37.

19. Schnek, A. G., and W. A. Schroeder. 1961. The relation between the minor components of whole normal human adult hemoglobin as isolated by chromatography and starch block electrophoresis. J. Am. Chem. Soc. 83:1472-1478.

20. Abraham, E. C., T. A. Huff, N. D. Cope, J. B. Wilson, Jr., E. D. Bransome, Jr., and T. H. J. Huisman. 1978. Determination of the glycosylated hemoglobins ( $\mathrm{Hb} \mathrm{A_{1 }}$ ) with a new microcolumn procedure. Suitability of the technique for assessing the clinical management of diabetes mellitus. Diabetes. 27:931-937.

21. Allison, T., C. C. Wood, and W. R. Goff. 1983. Brain stem auditory, pattern-reversal visual, and short-latency somatosensory evoked potentials: latencies in relation to age, sex, and brain and body size. Electroencephalogr. Clin. Neurophysiol. 55:619-636.

22. Sundlöf, G., and B. G. Wallin. 1978. Human muscle nerve sympathetic activity at rest. Relationship to blood pressure and age. J. Physiol. (Lond.). 274:621-637.

23. Gibbons, J. D. 1976. Nonparametric Methods for Quantitative Analysis. Holt, Rinehart and Winston, New York. 136-137, 171-173, 289-294.

24. Fouad, F. M., R. C. Tarazi, C. M. Ferrario, S. Fighaly, and C. Alicandri. 1984. Assessment of parasympathetic control of heart rate by a noninvasive method. Am. J. Physiol. 246:H838-H842.

25. Wallin, B. G., G. Sundlöf, B.-M. Eriksson, P. Dominiak, H. Grobecker, and L. E. Linblad. 1981. Plasma noradrenaline correlates to sympathetic muscle nerve activity in normotensive man. Acta Physiol. Scand. 111:69-73.

26. Robertson, D., G. A. Johnson, R. M. Robertson, A. S. Nies, D. G. Shand, and J. A. Oates. 1979. Comparative assessment of stimuli that release neuronal and adrenomedullary catecholamines in man. Circulation. 59:637-643.

27. Öberg, B., and P. Thorén. 1972. Increased activity in left ventricular receptors during hemorrhage or occlusion of caval veins in the cat.-A possible cause of the vaso-vagal reaction. Acta Physiol. Scand. $85: 164-173$

28. Barcroft, H., O. G. Edholm, J. McMichael, and E. P. SharpeySchafer. 1944. Posthaemorrhagic fainting. Study by cardiac output and forearm flow. Lancet. 1:489-491.

29. Murray, R. H., L. J. Thompson, J. A. Bowers, and C. D. Albright. 1968. Hemodynamic effects of graded hypovolemia and vasodepressor syncope induced by lower body negative pressure. Am. Heart J. 76:799811.

30. Wallin, B. G., and G. Sundlöf. 1982. Sympathetic outflow to muscles during vasovagal syncope. J. Auton. Nerv. Syst. 6:287-291.

31. Chen, R. Y. Z., R. S. Matteo, F.-C. Fan, G. B. Schuessler, and S. Chien. 1982. Resetting of baroreflex sensitivity after induced hypotension. Anesthesiol. 56:29-35.

32. Lin, M.-S., J. L. McNay, A. M. M. Shepherd, and T. K. Keeton. 1983. Effects of hydralazine and sodium nitroprusside on plasma catecholamines and heart rate. Clin. Pharmacol. Ther. 34:474-480.

33. Dorward, P. K., W. Riedel, S. L. Burke, J. Gipps, and P. I. Korner. 1985. The renal sympathetic baroreflex in the rabbit. Arterial and cardiac baroreceptor influences, resetting, and effect of anesthesia. Circ. Res. 57: 618-633.

34. Clarke, B. F., D. J. Ewing, and I. W. Campbell. 1979. Diabetic autonomic neuropathy. Diabetologia. 17:195-212.

35. Grubeck-Loebenstein, B., H. Vierhapper, W. Waldhäusl, A. Korn, M. Graf, and S. Panzer. 1982. Adrenergic mechanisms and blood pressure regulation in diabetes mellitus. Klin. Wochenschr. 60:823-828.

36. Lake, C. R., B. Chernow, D. S. Goldstein, D. G. Glass, M. Coleman, and M. G. Ziegler. 1984. Plasma catecholamine levels in normal subjects and in patients with secondary hypertension. Fed. Proc. 43:5256.

37. Cryer, P. E., A. B. Silverberg, J. V. Santiago, and S. D. Shah. 1978. Plasma catecholamines in diabetes. The syndromes of hypoadrenergic and hyperadrenergic postural hypotension. Am. J. Med. 64: 407-416.

38. Neubauer, B., and N. J. Christensen. 1976. Norepinephrine, epinephrine, and dopamine contents of the cardiovascular system in longterm diabetics. Diabetes. 25:6-10.

39. Goldstein, D. S. 1983. Arterial baroreflex sensitivity, plasma catecholamines, and pressor responsiveness in essential hypertension. Circulation. 68:234-240.

40. Folkow, B., M. Hallbäck, Y. Lundgren, R. Sivertsson, and L. Weiss. 1973. Importance of adaptive changes in vascular design for establishment of primary hypertension, studied in man and in spontaneously hypertensive rats. Circ. Res. (Supp. 1)32 and 33:I2-I13.

41. Christlieb, A. R., H.-U. Janka, B. Kraus, R. E. Gleason, E. A. Icasas-Cabral, L. M. Aiello, B. V. Cabral, and A. Solano. 1976. Vascular reactivity to angiotension II and to norepinephrine in diabetic subjects. Diabetes. 25:268-274.

42. Kontos, H. A., D. W. Richardson, and J. E. Norvell. 1975. Norepinephrine depletion in idiopathic orthostatic hypotension. Ann. Intern. Med. 82:336-341.

43. Ziegler, M. G., C. R. Lake, and I. J. Kopin. 1977. The sympatheticnervous-system defect in primary orthostatic hypotension. $N$. Engl. J. Med. 296:293-297.

44. Donald, M. W., D. L. W. Erdahl, D. H. C. Surridge, T. N. Monga, J. S. Lawson, C. E. Bird, and F. J. J. Letemendia. 1984. Functional correlates of reduced central conduction velocity in diabetic subjects. Diabetes. 33:627-633.

45. Duchen, L. W., A. Anjorin, P. J. Watkins, and J. D. Mackay. 1980. Pathology of autonomic neuropathy in diabetes mellitus. Ann. Intern. Med. 92:301-303. 